\title{
Self-supervised learning for random noise suppression in seismic data
}

Claire Birnie, Matteo Ravasi and Tariq Alkhalifah, King Abdullah University of Science and Technology (KAUST)

\section{SUMMARY}

A staunch companion to seismic signals, noise consistently hinders processing and interpretation of seismic data. Borrowing ideas from the field of computer vision, we propose the use of self-supervised deep learning for the task of random noise suppression. These techniques require no clean training data and therefore remove any requirement of pre-cleaning of field data or the generation of realistic synthetic datasets for training purposes. Through the use of blind-spot networks, we show that self-supervised Noise2 Void (N2V) procedure can be adapted to the seismic context, and trained solely on noisy data. An initial validation performed on a synthetic dataset corrupted by additive, white, Gaussian noise confirms that $\mathrm{N} 2 \mathrm{~V}$ can be trained to accurately separate the correlated seismic signal from the uncorrelated noise. Furthermore, when correlated and random noise are both present in the data, whilst the model cannot remove the majority of the correlated noise, a portion of it is suppressed alongside the random noise. Finally, the network is validated on a field dataset that is heavily contaminated with strong random noise caused by the surface conditions. The N2V denoising approach is shown to drastically reduce the random noise in the data. Through these examples, we have validated the effectiveness of blind-spot networks on highly oscillating signals, such as seismic data. This pave the way for the application of other self-supervised procedures to seismic data that go beyond the assumption of statistically independent noise.

\section{INTRODUCTION}

Noise is an ever-present challenge in the processing and interpretation of seismic data. As such, noise attenuation methods are employed at different stages of processing with the aim of enhancing the coherent component of the data over the random one. Random noise attenuation is generally carried out in the original $t-x$ domain by means of median filtering (Stewart, 1985), in the f-x domain using prediction error filters (Canales, 1984), or in other transformed domains such as Wavelets (Zhang and Ulrych, 2003) or Curvelets (Neelamani et al., 2008) where signal and noise show minimal overlap.

Recently, the success of deep learning has led to the development of a number of noise suppression procedures for seismic applications, which range from random noise suppression (Liu et al., 2018), groundroll removal (Kaur et al., 2020), and source deghosting (Vrolijk and Blacquière, 2021). A key limitation of these deep learning procedures is the fact that they require supervision, i.e., noise-free labels. Therefore, a noise-free version of the seismic data must be available alongside with the noisy training data. Currently, two approaches have been proposed to circumvent this unfeasible requirement: a conventional suppression technique is applied on the training dataset to create the 'noise-free' labels, or synthetic data are generated for training purposes. In the former approach the performance of the neural network is evidently limited by that of the suppression procedure used to create the training data (results in the training of a model to mimic the performance of the conventional suppression procedure), whilst the latter approach requires the signal and noise to been realistically modelled in the generation of the synthetic data, clearly a non-trivial task (Birnie et al., 2016).

In this work, we illustrate how self-supervised neural networks can be used to remove the requirement of providing a noisefree training label set. Initially illustrated on synthetic data, the self-supervised network is shown to be a powerful noise suppression procedure aiding down the line tasks, such as poststack inversion. Finally, the approach is applied to a post-stack time migrated data from a land seismic survey that is heavily contaminated with random noise caused by the surface conditions.

\section{THEORY: SELF-SUPERVISED DENOISING}

The requirement for pairs of noisy and clean images for training denoising procedures is often unfeasible, particularly in the seismic domain where such clean (i.e. noise-free) datasets are impossible to collect or re-create by by means of state-of-theart denoising procedures. In other words, there is no perfect denoising technique available that will remove all the noise from a field seismic recording.

Over the last few years, significant effort has been devoted to developing denoising procedures that do not require a clean image for training; such methods have already shown great promise both in the field of computer vision when dealing with natural images and in the field of medical microscopy. Noise2Noise (Lehtinen et al., 2018) is the first of such selflearning techniques, which takes two images with different noise realizations as input; by assuming that the signal is constant between the two images, a network is trained to learn the mapping between them. Provided that the network is not overfitting, this acts as a natural denoiser. To overcome the limitation of requiring two images of the same scene, Krull et al. (2019) proposed Noise2Void (N2V), which works on a single image by utilising so-called blind-spot networks. These algorithms are based on the assumption that the signal is spatially correlated and they can successfully learn to remove pixel-wise independent noise.

$\mathrm{N} 2 \mathrm{~V}$ works by replacing a set of non-adjacent pixels $\mathscr{J}$ in an image $x$ with randomly selected pixels $\mathscr{g}^{\prime}$ that pertain to the receptive field of the chosen network, as illustrated in Figure 1. The corrupted image becomes the input to a standard NN architecture denoted as $f_{\theta}$ where $\theta$ refers to the trainable parameters (in our case a U-Net of Ronneberger et al. (2015) was used), whilst the original noisy image is used as the target. As opposed to standard NN image processing tasks, the loss func- 


\section{Self-supervised learning for noise suppression}
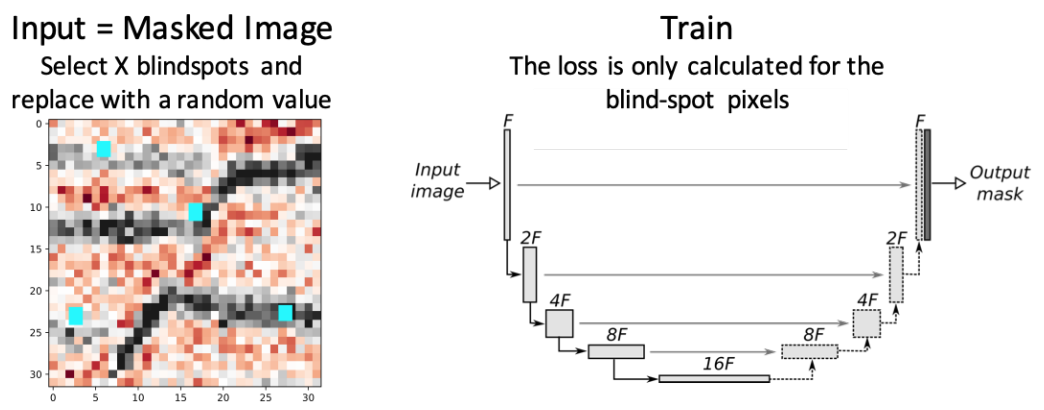

Target $=$ Noisy Image

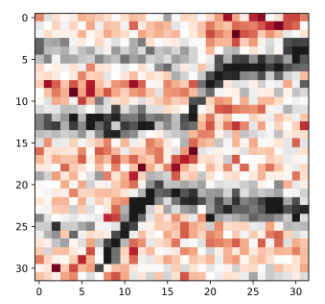

Figure 1: Schematic of the application of N2V on a seismic dataset. The blue squares in the input image illustrate the randomly selected pixels whose values are replaced by that of a pixel within their receptive field.

tion is not computed on every pixel in the input data, instead it is only evaluated for the blind-spotted pixels, i.e., those that were corrupted in the input image:

$$
\hat{\boldsymbol{\theta}}=\underset{\theta}{\operatorname{argmin}} \frac{1}{N_{S}} \sum_{i=1}^{N_{s}}\left\|\mathbb{I}_{\mathscr{J}}\left(f_{\theta}\left(\mathbb{I}_{\mathscr{F}^{\prime} \rightarrow \mathscr{F}}\left(x_{i}\right)\right)\right)-\mathbb{I}_{\mathscr{G}}\left(x_{i}\right)\right\|_{1}
$$

where $\mathbb{I}_{\mathscr{F}^{\prime} \rightarrow \mathscr{g}}$ applies the blind-spot operation, $\mathbb{I}_{\mathscr{g}}$ extracts the values of the input image at the pixels $\mathscr{J}$, and $N_{s}$ is the number of available training samples. In our experience, the meanabsolute error (i.e., L1 norm) has shown superior performance to the more commonly used mean-square error for seismic applications, and as such is used in this work.

\section{SYNTHETIC EXAMPLES}

The N2V approach is initially tested on a post-stack synthetic dataset modelled from SEG Hess VTI model to which we add White, Gaussian Noise (WGN), as illustrated in Figure 2(a) and (b), respectively. In order to mimic a 3D dataset which would provide multiple inlines or crosslines with varying noise realisations, 50 2D slices are created with varying levels of WGN for the N2V training. The dataset is then cropped into 32-by-32 pixel windows, with 5000 windows being used for training and 500 being retained for validation purposes. Similar to Krull et al. (2019), we mask $0.2 \%$ of the pixels within each window and use a 2-layer U-Net architecture trained over 100 epochs. Figure 2(c) illustrates the application of the trained model to the noisy dataset shown in Figure 2(b), while (d) and (e) highlight the difference between the denoised data and the noisy and noise-free datasets, respectively. Whilst a large amount of noise has been suppressed, as is apparent in Figure 2(d), Figure 2(e) reveals that some of the signal has also been partially damaged. Finally, the average amplitude spectra of the noise-free, noisy, and denoised datasets are shown in Figure 2(f). The spectrum of the denoised data very closely matches that of the original noise-free dataset, with a drastic reduction in the noise across all frequency bands.

To further assess the importance of the $\mathrm{N} 2 \mathrm{~V}$ denoising procedure, a spatially-regularized post-stack inversion (Ravasi and Birnie, 2021) is performed on both the noisy and denoised dataset as shown in Figure 2(h) and (i), respectively. Despite the estimated acoustic impedance model of the denoised data is still not identical to the true model, we observe a drastic improvement, both with respect to the Signal-to-Noise Ratio (SNR) and continuity of layers in comparison to the noisy inversion.

\section{Correlated Noise}

One of the assumptions of $\mathrm{N} 2 \mathrm{~V}$ is that noise must be statistically independent between neighbouring pixels. However, it is unlikely that noise in seismic data is ever truly uncorrelated (in both time and space). To test whether this assumption can be partly relaxed, we generate a second dataset where random noise is supplemented with correlated noise with a 5 pixel window, i.e. 5 steps in both space $(x)$ and time $(y)$, with a 40/60 ratio of random to correlated noise (Figure $3 \mathrm{a}$ ). To avoid overfitting, which would lead the model to learn to predict the noise as well as the signal, we reduce the number of training epochs from 100 to 10 and increase the number of blind-spots to $25 \%$ of the samples. By doing so, we introduce some inevitable cross-talk between the different receptive fields of the blindspots. As a consequence, each receptive field now contains more than one corrupted pixel that introduce additional randomnicity in the learning process. Similar to before, $500032-$ by-32 windows are used for training with a further 500 being retained for validation.

Figure 3(b) illustrates the denoised results, which show a significant improvement in the SNR however some correlated noise remains. Figure 3(c) shows the original correlated noise that was included into the noisy dataset whilst Figure 3(d) shows the difference between the denoised data and the clean wavefield data (Figure 2(a)). The difference in the noise amplitudes highlight that some correlated noise has been suppressed through the random noise suppression procedure. Nevertheless, similar to the WGN example in Figure 2, small signal leakage is observed, most notably around the central salt body.

\section{FIELD DATA EXAMPLE}

To conclude our analysis on the applicability of N2V for random noise suppression of seismic data, we apply it to a timemigrated land field dataset that is heavily contaminated by strong random noise (Liu and Chen, 2013). The original migrated 


\section{Self-supervised learning for noise suppression}

(a)

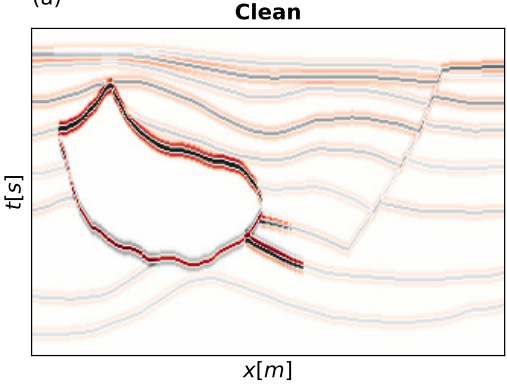

(d)

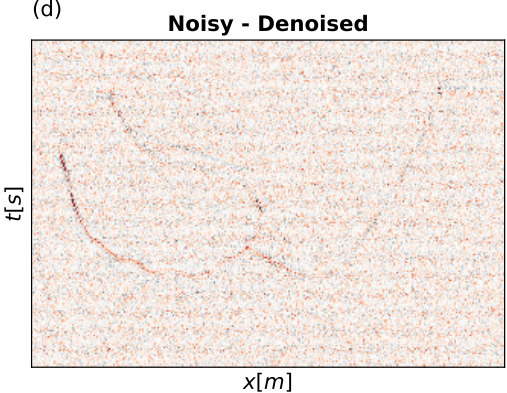

(g)

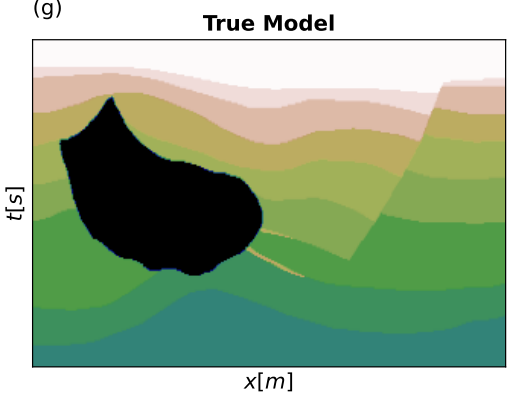

(b)

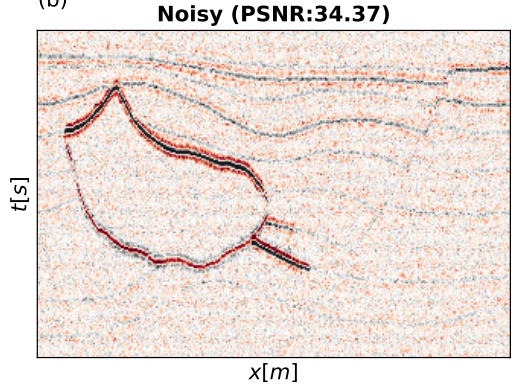

(e)

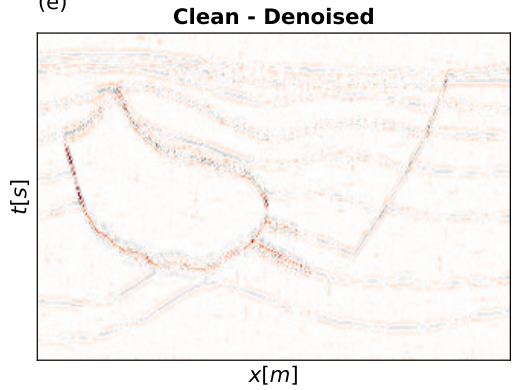

(h)

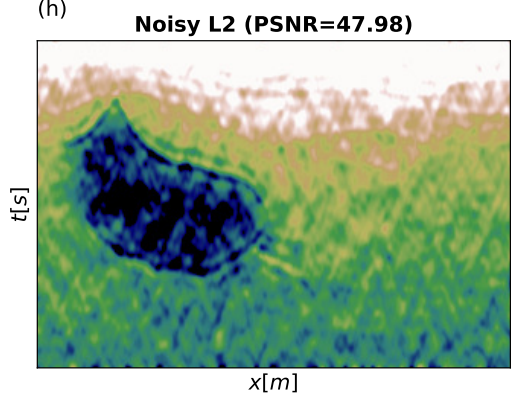

(c)

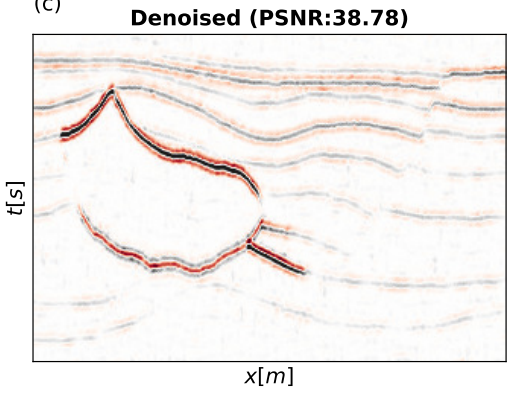

(f)

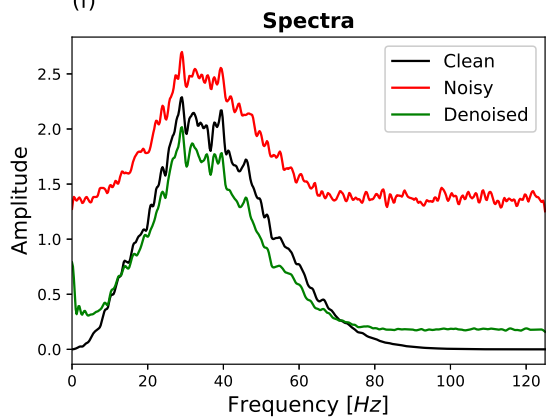

(i)

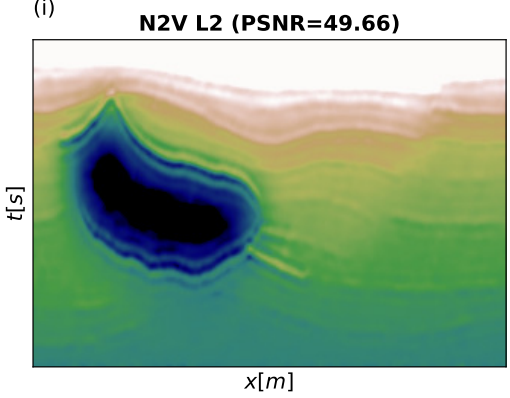

Figure 2: N2V self-learning denoising procedure applied to the Hess synthetic post-stack dataset containing a significant amount of random noise. (a) Noise-free data, (b) noisy data with additive WGN, (c) denoised data, (d) noise removed from the noisy image via the denoising procedure, (e) difference between the noise-free and denoised data, and (f) the different spectra per data panel in the top row. (g) Represents the true subsurface model whilst (h) and (i) illustrate the L2-regularised inversion result for the noisy and denoised data, respectively.

data is shown in Figure 4(a). Similar to the previous example, the hyperparameters of the network are tuned to avoid overfitting and compensate for the presence of some degree of correlation in the noise, which violated the $\mathrm{N} 2 \mathrm{~V}$ assumption that noise must be statistically independent. Due to the limited size of the dataset, data augmentation procedures - polarity flipping and window rotation - are employed to increase the volume of the available training data; as a result, after 3000 training samples with a window size of 32-by-32 are fed to the network. In this example, $25 \%$ of the samples within each window are masked and a 2-layer UNet is trained over 10 epochs. Figure $4(\mathrm{~b})$ portrays the denoised data, whilst three sub-areas are shown in panels $(\mathrm{c}, \mathrm{e}, \mathrm{g})$ for the original data and $(\mathrm{d}, \mathrm{f}, \mathrm{h})$ for the denoised data, respectively. Across almost the entire dataset, it is clear that the trained N2V model has successfully suppressed a large portion of the random noise that was plaguing the dataset.

\section{DISCUSSION AND CONCLUSIONS}

In this work, we have presented how self-learning algorithms such as N2V can be adapted for and applied to seismic data for denoising purposes. Through an illustration on synthetic data, $\mathrm{N} 2 \mathrm{~V}$ is shown to successfully suppress random noise, increasing the SNR and resulting in improved post-stack inversion. However, N2V relies on the assumption that noise is statistically independent between samples, which is often not the case for seismic data. Through an example containing correlated noise we showed that whilst N2V cannot fully suppress the correlated noise, a portion of it is still suppressed alongside the random component of the noise. We conclude this work by showing the applicability of $\mathrm{N} 2 \mathrm{~V}$ to a field dataset contaminated by strong random noise. $\mathrm{N} 2 \mathrm{~V}$ removes the requirement of supervised learning for a clean target dataset. As such, not only is the resulting model not biased by the performance of a 


\section{Self-supervised learning for noise suppression}
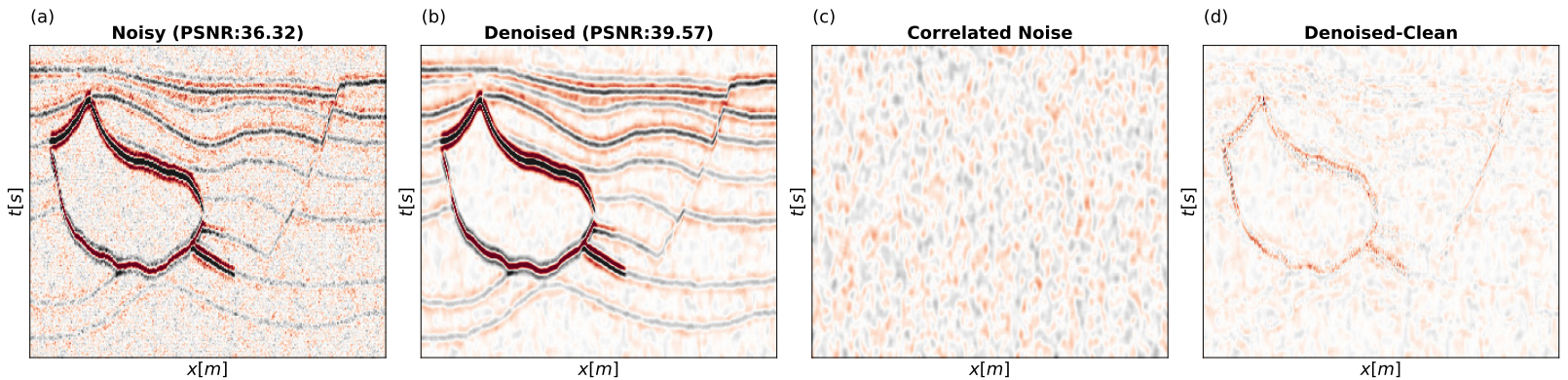

Figure 3: N2V self-learning denoising procedure applied to the Hess synthetic post-stack dataset containing $40 \%$ random noise and $60 \%$ noise correlated over 5 time-space samples. (a) Noisy data, (b) denoised data, (c) correlated noise component of the noisy data, and (d) difference between the denoised data and the true noise-free data.
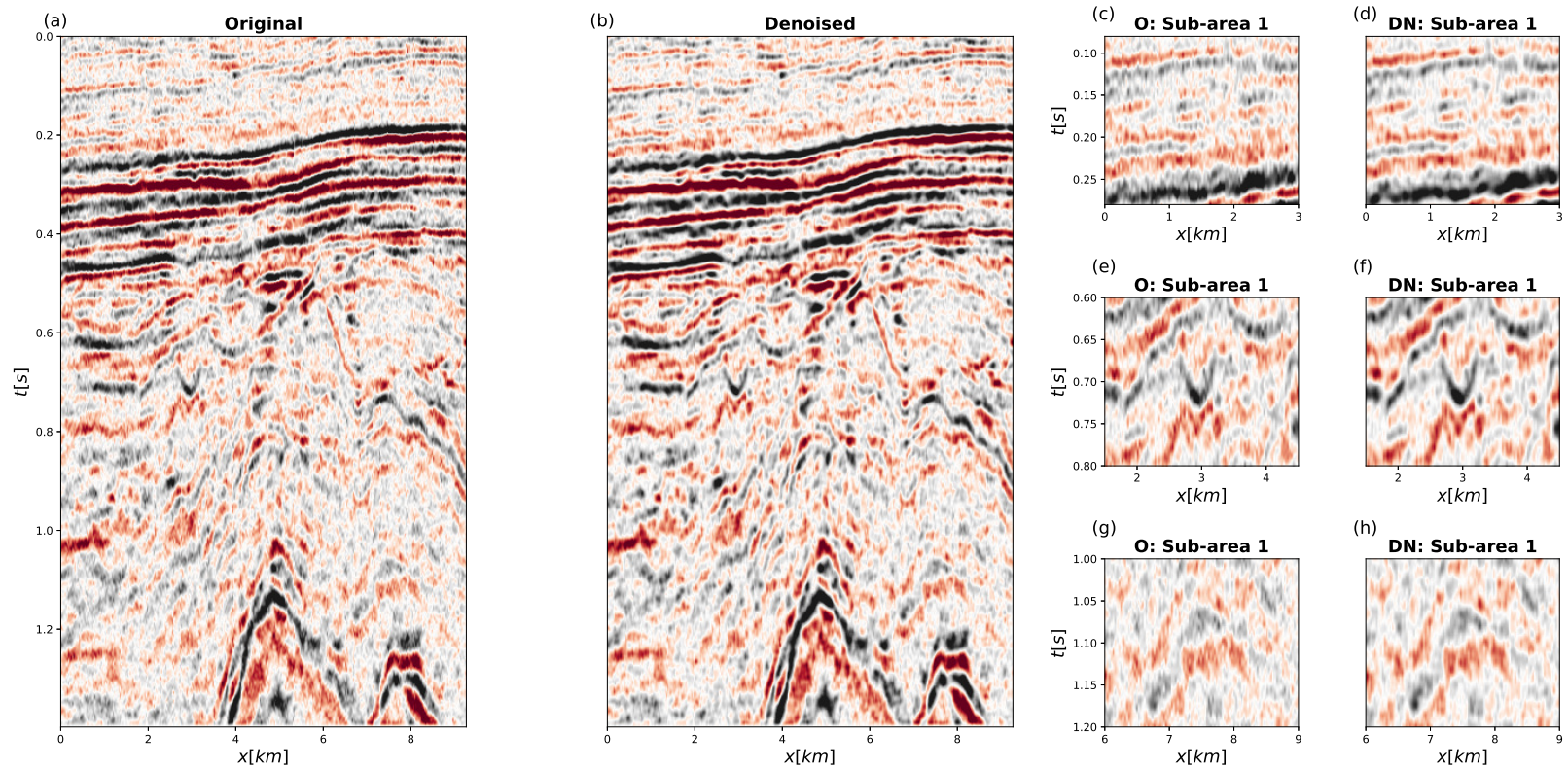

(f)
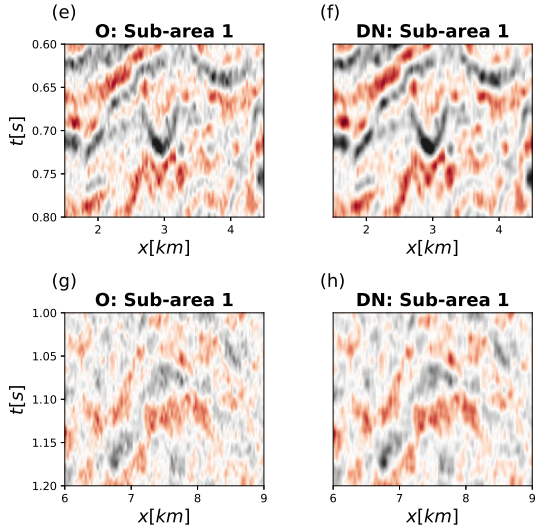

Figure 4: N2V self-learning denoising procedure applied to a noisy land dataset contaminated with strong surface noise. The right plots illustrate different areas of the dataset zoomed-in to highlight the SNR improvements achieved via denoising with the original data shown in $(\mathrm{c}, \mathrm{e}, \mathrm{g})$ and the respective denoised sections shown in $(\mathrm{d}, \mathrm{f}, \mathrm{h})$.

denoising algorithm required to make the clean target images, but it also significantly speeds up the denoising workflow by completely removing the costly training dataset creation stage.

Despite its successful application to field data, significant parameter changes were required to adapt the approach to handling seismic data. This is likely because the seismic data violates the $\mathrm{N} 2 \mathrm{~V}$ assumption of statistical independence between noise samples. To counteract this, a successful strategy included reducing the number of training epochs by a factor of 10 and increasing the number of masked pixels by a factor of 100 , in comparison to the application of $\mathrm{N} 2 \mathrm{~V}$ on both standard images and microscopy data. Whilst we have shown that with carefully selected hyperparameters $\mathrm{N} 2 \mathrm{~V}$ can be successfully applied to seismic data, a more suitable alternative may be represented by N2V's successor: Structured Noise2Void (StructN2V - Broaddus et al. (2020)). As opposed to random pixel masking, StructN2V employs a structured noise mask designed to adapt to the structure of the noise (i.e., correlation length and directionality). By removing the constraint of independent noise, StructN2V has the potential to improve various seismic processing step through self-supervised denoising of correlated data. Future work will focus on considering how optimal masks can be generated for StructN2V such that it too can be adapted to be applicable for seismic data.

\section{ACKNOWLEDGMENTS}

The authors thank A. Krull, T.-O. Buchholz, and F. Jug for open-sourcing their TensorFlow implementation of Noise2 Void. For computer time, this research used the resources of the Supercomputing Laboratory at King Abdullah University of Science Technology (KAUST) in Thuwal, Saudi Arabia. 


\section{REFERENCES}

Birnie, C., K. Chambers, D. Angus, and A. L. Stork, 2016, Analysis and models of pre-injection surface seismic array noise recorded at the aquistore carbon storage site: Geophysical Journal International, 206, 1246-1260.

Broaddus, C., A. Krull, M. Weigert, U. Schmidt, and G. Myers, 2020, Removing structured noise with self-supervised blind-spot networks: 2020 IEEE 17th International Symposium on Biomedical Imaging (ISBI), IEEE, 159-163.

Canales, L., 1984, Random noise reduction: SEG expanded abstracts:54th Annual international meeting, 525-527.

Kaur, H., S. Fomel, and N. Pham, 2020, Seismic groundroll noise attenuation using deep learning: Geophysical Prospecting, 68, 2064-2077.

Krull, A., T.-O. Buchholz, and F. Jug, 2019, Noise2voidlearning denoising from single noisy images: Proceedings of the IEEE/CVF Conference on Computer Vision and Pattern Recognition, 2129-2137.

Lehtinen, J., J. Munkberg, J. Hasselgren, S. Laine, T. Karras, M. Aittala, and T. Aila, 2018, Noise2noise: Learning image restoration without clean data: arXiv preprint arXiv:1803.04189.

Liu, D., W. Wang, W. Chen, X. Wang, Y. Zhou, and Z. Shi, 2018, Random noise suppression in seismic data: What can deep learning do?: SEG Technical Program Expanded Abstracts 2018, Society of Exploration Geophysicists, 20162020.

Liu, G. C., and X. H. Chen, 2013, Noncausal f-x-y regularized nonstationary prediction filtering for random noise attenuation on 3d seismic data: Journal of Applied Geophysics, 93, 60-66.

Neelamani, R., A. I. Baumstein, D. G. Gillard, M. T. Hadidi, and W. L. Soroka, 2008, Coherent and random noise attenuation using the curvelet transform: The Leading Edge, 27.

Ravasi, M., and C. E. Birnie, 2021, A joint inversionsegmentation approach to assisted seismic interpretation: arXiv preprint arXiv:2102.03860.

Ronneberger, O., P. Fischer, and T. Brox, 2015, U-net: Convolutional networks for biomedical image segmentation: International Conference on Medical image computing and computer-assisted intervention, Springer, 234-241.

Stewart, R. R., 1985, Median filtering: Review and a new f/k analogue design: Journal of the Canadian Society of Exploration Geophysicists, 21, 54-63.

Vrolijk, J.-W., and G. Blacquière, 2021, Source deghosting of coarsely sampled common-receiver data using a convolutional neural network: Geophysics, 86, V185-V196.

Zhang, R., and T. Ulrych, 2003, Physical wavelet frame denoising: Geophysics, 68, 225-231. 